\title{
IncRNA WT1-AS is upregulated in osteoporosis and regulates the apoptosis of osteoblasts by interacting with p53
}

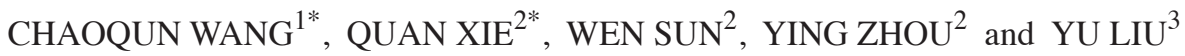 \\ ${ }^{1}$ Department of Nuclear Medicine, Hainan General Hospital, Haikou, Hainan 570311; ${ }^{2}$ Department \\ of Nuclear Medicine; ${ }^{3}$ Cancer Institute Of Hainan Medicail University, The First Affiliated \\ Hospital of Hainan Medical University, Haikou, Hainan 570100, P.R. China
}

Received May 1, 2019; Accepted January 17, 2020

DOI: $10.3892 /$ etm.2021.10166

\begin{abstract}
In cervical cancer, cellular tumor antigen p53 (p53) interacts with long non-coding WT1 antisense RNA (WT1-AS) and this protein serves an important role in osteoporosis. The present study aimed to investigate the role of WT1-AS in osteoporosis. WT1-AS was upregulated in the plasma of patients with osteoporosis and was positively correlated with p53 expression. Altered expression of WT1-AS and p53 separated patients with osteoporosis from healthy controls. Expression levels of WT1-AS and p53 decreased with prolonged treatment. In osteoblasts, WT1-AS overexpression resulted in increased p53 expression, while WT1-AS small interfering RNA (siRNA) silencing resulted in decreased p53 expression. In addition, WT1-AS overexpression resulted in increased apoptosis rate, while WT1-AS siRNA silencing resulted in decreased apoptosis rate in osteoblasts. p53 overexpression attenuated the effects of WT1-AS siRNA silencing on cell apoptosis. Therefore, WT1-AS was upregulated during osteoporosis and regulated the apoptosis of osteoblasts by interacting with p53.
\end{abstract}

\section{Introduction}

As a common type of skeletal disorder, osteoporosis is characterized by abnormal bone architecture and low bone mineral density, resulting in increased risk of fracture (1). Osteoporosis was once considered an inevitable disorder in the elderly population. Recently, multiple prevention and treatment approaches have been developed for osteoporosis (2). However, with the growth of the aging population, the incidence of osteoporosis is increasing worldwide, alongside increased treatment

Correspondence to: Dr Ying Zhou, Department of Nuclear Medicine, The First Affiliated Hospital of Hainan Medical University, 31 Longhua Road, Haikou, Hainan 570100, P.R. China E-mail: tkfqtau642923@126.com

\section{${ }^{*}$ Contributed equally}

Key words: osteoporosis, WT1 antisense RNA, cellular tumor antigen p53, osteoblast costs (3). Therefore, novel therapeutic approaches with higher efficiencies are required to improve the treatment outcomes of osteoporosis. Osteoblasts and osteoclasts serve critical roles in bone formation and resorption, respectively $(4,5)$. However, molecular mechanisms that are associated with this disease remain poorly understood $(4,5)$, leading to difficulties in the development of novel therapeutic regimens.

p53 signaling is a well-studied pathway that plays pivotal roles in diverse cellular processes, such as cell cycle progression, genomic stability and cell apoptosis (6-8). p53 can regulate the apoptosis of osteoblastic cells (9), which plays a key role in the pathogenesis of osteoporosis (10). Inhibition of p53 suppresses the apoptosis of osteoblasts, thereby contributing to recovery from osteoporosis (11). It has been reported that the development and progression of osteoporosis also requires the involvement of long (>200 nt) non-coding RNAs (lncRNAs), which participates in diverse biological processes by regulating gene expression (12). It has been previously reported that WT1-AS can upregulate $\mathrm{p} 53$ in cervical cancer to inhibit cancer progression (13). Therefore, WT1-antisense RNA (WT1-AS) may also interact with p53 to participate in the development of osteoporosis. The present study was performed to explore the possible interaction between WT1-AS and p53 in osteoporosis.

\section{Materials and methods}

Research subjects. The present study included 60 patients with osteoporosis (23 males and 37 females; age range 33-66 years; mean age, $49.2 \pm 6.1$ years) and 60 healthy volunteers (23 males and 37 females, 32-66 years; mean age, 49.6 \pm 6.3 years). All patients and healthy volunteers were admitted to the First Affiliated Hospital of Hainan Medical College between March 2015 and March 2018. The inclusion criteria of patients were as follows: i) Newly diagnosed cases; and ii) no initiated therapies. The exclusion criteria of patient were as follows: i) Recurrent cases; and ii) complications with other bone disorders or other types of diseases. All patients were informed of the experimental principles, and gave their signed informed consent. The Ethics Committee of the First Affiliated Hospital of Hainan Medical College approved this study prior to the admission of subjects. No significant differences in age, gender, body mass index, or smoking and drinking history were indicated between the two groups (data not shown). The T-score 
was calculated using the following formula: $\mathrm{T}$-score $=($ bone mineral density-reference bone mineral density)/reference standard deviation (14). The T-score of the patients ranged from -2.5 to -4.7 (mean score, $-3.3 \pm 0.4$ ), while the T-score for healthy volunteers ranged from -0.9 to 3.3 (mean score, $1.3 \pm 0.5$ ). T-score was significantly lower in patients compared with controls (data not shown). The disease duration of patients ranged from 2.2 to 13.8 years, with a mean of $8.1 \pm 2.4$ years.

Patient treatment and plasma sample preparation. All 60 patients with osteoporosis were treated with bisphosphonates, including alendronate, risedronate, zoledronic acid and ibandronate. Estrogen was only used in females. Bisphosphonates attenuate bone loss, and estrogen was used in female patient to control postmenopausal symptoms (15). Drug doses were determined according to patients' health conditions, disease severity and mid-term treatment outcomes. Blood $(5 \mathrm{ml})$ was extracted from each healthy volunteer and patient under fasting conditions prior to therapy initiation (1-3 days). The same amount of fasting blood was also extracted from each patient with osteroporosis at 3 months after therapy initiation. Blood samples were centrifuged at 1,200 x g (room temperature) for $15 \mathrm{~min}$ to prepare plasma samples.

Transient transfection of osteoblasts. In vitro experiments were performed using primary osteoblasts purchased from Sigma-Aldrich; Merck KGaA. Primary osteoblasts were cultivated in Osteoblast Growth Medium (PromoCell GmbH) at $37^{\circ} \mathrm{C}$. All subsequent experiments were performed using cells from passage 4 or 5. WT1-AS and p53 expression vectors (pcDNA3.1) and empty pcDNA3.1 vector, as well as small interfering RNA (siRNA) negative control and WT1-AS siRNA, were synthesized by Guangzhou RiboBio Co., Ltd. Osteoblasts $\left(1 \times 10^{6}\right)$ were transfected with $10 \mathrm{nM}$ WT1-AS or p53 expression vector, $10 \mathrm{nM}$ empty pcDNA3.1 vector (negative control, NC group), 40 nM WT1-AS siRNA, or 40 nM siRNA NC (NC group). Non-transfected osteoblasts acted as the control (C) group. Osteoblast RNA and protein were harvested at $24 \mathrm{~h}$ post-transfection for use in subsequent experiments.

ELISA. Human p53 ELISA kit (cat. no. ab46067; Abcam) was used to measure levels of p53 in plasma samples. All steps were performed following manufacturer's instructions. All plasma levels of p53 were expressed as pg/ml.

$R N A$ extraction and reverse transcription-quantitative $P C R$ $(R T-q P C R)$. A total of $1 \times 10^{6}$ osteoblasts and $2 \mathrm{ml}$ of plasma was mixed with $1 \mathrm{ml}$ of RNAzol (Sigma-Aldrich; Merck KGaA) to extract total RNAs. DNase I (Invitrogen; Thermo Fisher Scientific, Inc.) was used to digest genomic DNA in all RNA samples at $37^{\circ} \mathrm{C}$ for $1 \mathrm{~h}$. Following that, a qScript cDNA Synthesis kit (Quantabio) was used to perform RT $\left(25^{\circ} \mathrm{C}\right.$ for $10 \mathrm{~min}, 55^{\circ} \mathrm{C}$ for $20 \mathrm{~min}$ and $85^{\circ} \mathrm{C}$ for $10 \mathrm{~min}$ ). qPCR reaction mixtures were prepared using a KAPA SYBR FAST qPCR Master Mix kit (Roche Diagnostics). GAPDH was used as an endogenous control. Primer sequences were: WT1-AS, 5'-GCC TCTCTGTCCTCTTCTTTG-3' (forward), 5'-GCTGT GAGTC CTGGTGCTTA-3' (reverse); GAPDH, 5'-TTGGCATCGTTG AGGGTC-3' (forward), 5'-AGTGGGAACACGGAAAGC-3' (reverse); P53, 5'-AGAGTCTATAGGCCCACCCC-3' (forward),
Table I. Association between patients' clinical data and WT1-AS and p53 expression.

A, WTI-AS

\begin{tabular}{lccc}
\hline \multicolumn{3}{c}{ Expression } & \\
\cline { 2 - 3 } Index & High $(\mathrm{n}=30)$ & Low $(\mathrm{n}=30)$ & P-value \\
\hline Age & & & $>0.05$ \\
$\geq 50$ years & 14 & 15 & \\
$<50$ years & 16 & 15 & $>0.05$ \\
Sex & & & \\
Male & 13 & 10 & $>0.05$ \\
Female & 17 & 20 & \\
Smoking & & & $<0.001$ \\
Yes & 11 & 10 & \\
No & 19 & 20 & \\
Disease duration & & & \\
$\geq 8$ years & 22 & 6 & \\
$<8$ years & 8 & 24 & \\
T score & & 6 & \\
$\geq-3.5$ & 19 & & \\
\hline
\end{tabular}

B, p53

\begin{tabular}{lccc}
\hline \multicolumn{3}{c}{ Expression } & \\
\cline { 2 - 3 } Index & High $(\mathrm{n}=30)$ & Low $(\mathrm{n}=30)$ & P-value \\
\hline Age & & & $>0.05$ \\
$\geq 50$ years & 14 & 15 & \\
$<50$ years & 16 & 15 & \\
Sex & & & $>0.05$ \\
Male & 13 & 10 & \\
Female & 17 & 20 & \\
Smoking & & & \\
Yes & 11 & 10 & \\
No & 19 & 20 & $<0.001$ \\
Disease duration & & & \\
$\geq 8$ years & 22 & 6 & 0.001 \\
$<8$ years & 8 & 24 & \\
T score & & & \\
$\geq-3.5$ & 19 & 6 &
\end{tabular}

WT1-AS, long non-coding WT1 antisense RNA; p53, cellular tumor antigen $\mathrm{p} 53$.

5'-GCTCGACGCTAGGATCTGAC-3' (reverse). PCR cycling conditions were: $95^{\circ} \mathrm{C}$ for $1 \mathrm{~min}$, followed by 40 cycles of $95^{\circ} \mathrm{C}$ for $10 \mathrm{sec}$ and $58^{\circ} \mathrm{C}$ for $50 \mathrm{sec}$. The expression levels of WT1-AS and p53 mRNA were normalized using the $2^{-\Delta \Delta \mathrm{Cq}}$ method (16).

Western blot analysis. Osteoblasts were lysed with $1 \mathrm{ml}$ RIPA buffer (Guangzhou RiboBio Co., Ltd.) to extract total 
A

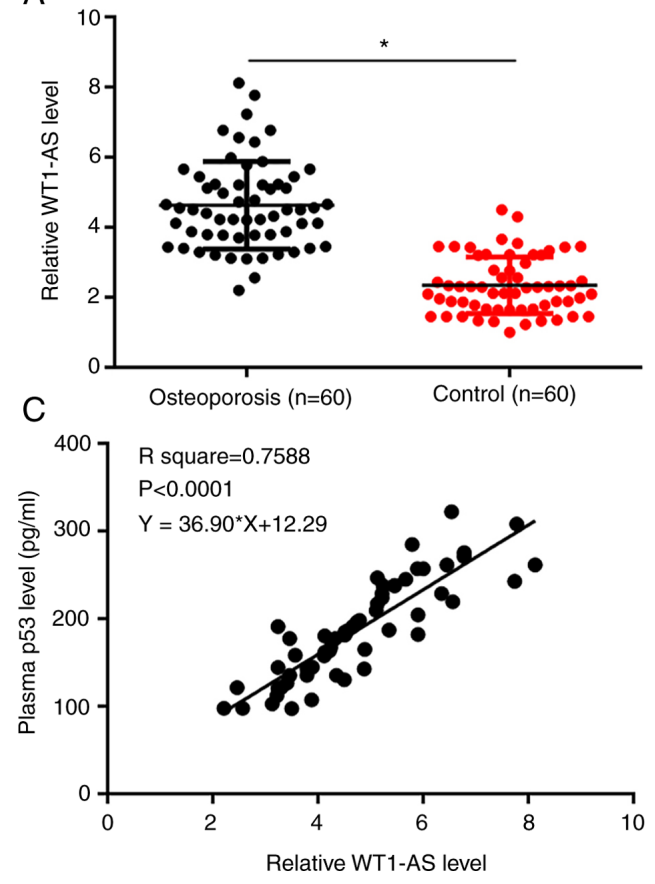

B
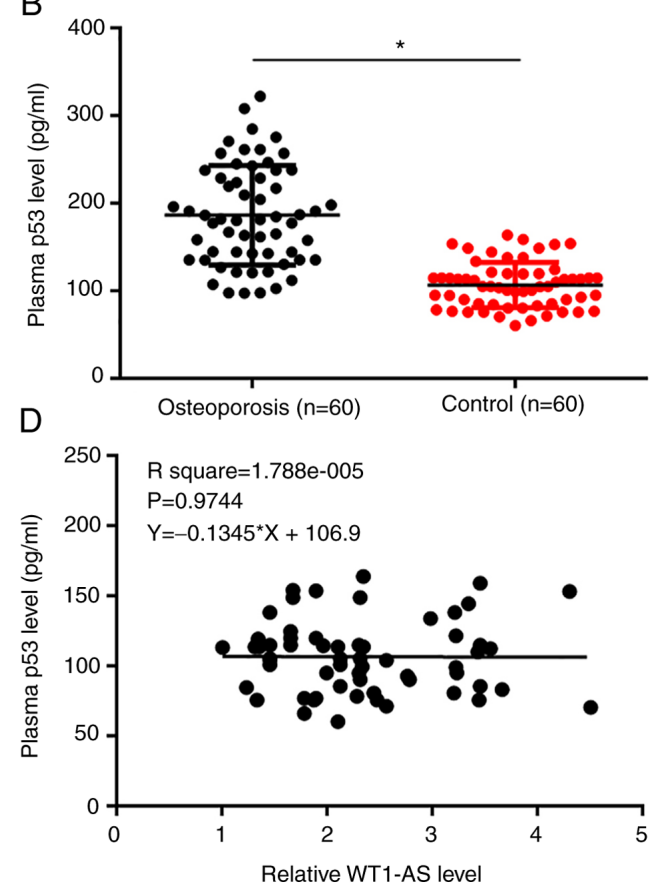

Figure 1. WT1-AS and p53 expression levels are positively correlated in patients with osteoporosis. Plasma levels of (A) WT1-AS and (B) p53 in plasma were measured using ELISA. Data between osteoporosis and control groups were compared by performing an unpaired t-test. Correlations between WT1-AS and p53 in (C) patients with osteoporosis and (D) healthy controls were analyzed using Pearson's correlation coefficient. ${ }^{*} \mathrm{P}<0.05$. WT1-AS, long non-coding WT1 antisense RNA; p53, cellular tumor antigen p53.

protein. Protein samples were quantified using a bicinchoninic acid kit (Sangon Biotech Co., Ltd.), followed by denaturation in boiling water for $5 \mathrm{~min}$. Electrophoresis was performed using $12 \%$ SDS-PAGE to separate proteins (30 $\mu \mathrm{g}$ per well) according to their molecular weights. Proteins were transferred to a PVDF membrane and blocking was carried out in $5 \%$ non-fat milk for $2 \mathrm{~h}$ at room temperature. Primary antibodies of rabbit GAPDH (1:1,200; cat. no. ab181602; Abcam) and p53 (1:1,200; cat. no. ab131442; Abcam) were used to incubate the membranes for $15 \mathrm{~h}$ at $4^{\circ} \mathrm{C}$. Horseradish peroxidase goat anti-rabbit (immunoglobulin G; 1:1,100; cat. no. ab6721; Abcam) secondary antibody was then used to blot the membranes further at room temperature for $2 \mathrm{~h}$. The ECL Chemiluminescence Detection kit (Sangon Biotech Co., Ltd.) was used to develop protein signals. Gray values were normalized using ImageJ version 1.46 (National Institutes of Health).

Cell apoptosis analysis. A total of $6 \times 10^{4}$ osteoblasts were mixed with $1 \mathrm{ml}$ serum-free aforementioned cell culture medium to prepare single-cell suspensions. Osteoblasts were cultivated at $37^{\circ} \mathrm{C}$ and $5 \% \mathrm{CO}_{2}$ in a 6 -well plate with $2 \mathrm{ml}$ of cell suspension in each well. Cells were incubated at $37^{\circ} \mathrm{C}$ for $48 \mathrm{~h}$ before being harvested and digested with $0.25 \%$ trypsin. Cells were subsequently stained using Annexin V-FITC (Thermo Fisher Scientific, Inc.) and propidium iodide (Thermo Fisher Scientific, Inc.) at $4^{\circ} \mathrm{C}$ for $20 \mathrm{~min}$ in dark. Apoptotic cells were analyzed using a flow cytometer. Data were processed using FCSalyzer Version 0.9.18 (SourceForge; DHI Group, Inc.).

Statistical analysis. The mean $\pm \mathrm{SD}$ values of three biological replicates of each experiment were calculated and used for all comparisons. Statistical power was calculated using Origin software version 10 (OriginLab Corp.) and a statistical power of $\sim 0.9$ was obtained. Differences between patients and controls were measured using an unpaired t-test. Differences between two time points in the patient group were analyzed using a paired t-test. Differences among different cell transfection groups were investigated using a one-way ANOVA followed by Tukey's test. Correlations were analyzed using Pearson's correlation coefficient. A receiver operating characteristic (ROC) curve was plotted for diagnostic analysis. Patients with disease are grouped in the true positive class and healthy controls are grouped in the true negative class. Patients were divided into high and low WT1-AS/p53 level groups $(n=30)$, using the mean expression levels of WT1-AS/p53 in osteoporosis as a cutoff score. Any association between patients' clinical data and WT1-AS/p53 expression was analyzed using a $\chi^{2}$ test. $\mathrm{P}<0.05$ was considered to indicate a statistically significant difference.

\section{Results}

WT1-AS and 53 levels are positively correlated in patients with osteoporosis. WT1-AS and p53 plasma levels were measured. Plasma levels of WT1-AS (Fig. 1A) and p53 (Fig. 1B) were significantly higher in patients with osteoporosis compared with the control group $(\mathrm{P}<0.05)$. Correlations between WT1-AS and p53 were analyzed using Pearson's correlation coefficient. WT1-AS and p53 levels were significantly and positively correlated in patients with osteoporosis (Fig. 1C, $\mathrm{P}<0.0001$ ), but not in healthy controls (Fig. 1D, $\mathrm{P}=0.9744)$. The T-score of the patients ranged from -2.5 to $-4.7 \cdot \chi^{2}$ test revealed that expression levels of WT1-AS and p53 were not associated with patients' age, sex and smoking habits, but were closely associated with T-score and disease duration (Table I). 

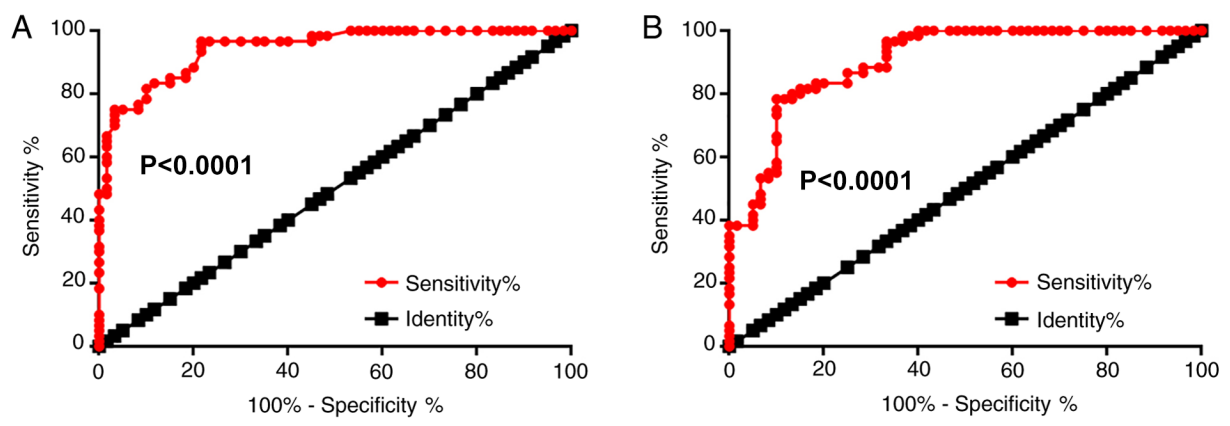

Figure 2. Altered expression levels of WT1-AS and p53 separate osteoporosis patients from healthy controls. The potential application of plasma (A) WT1-AS and (B) p53 for the diagnosis of osteoporosis was explored by plotting a receiver operating characteristic curve. WT1-AS, long non-coding WT1 antisense RNA; p53, cellular tumor antigen p53.
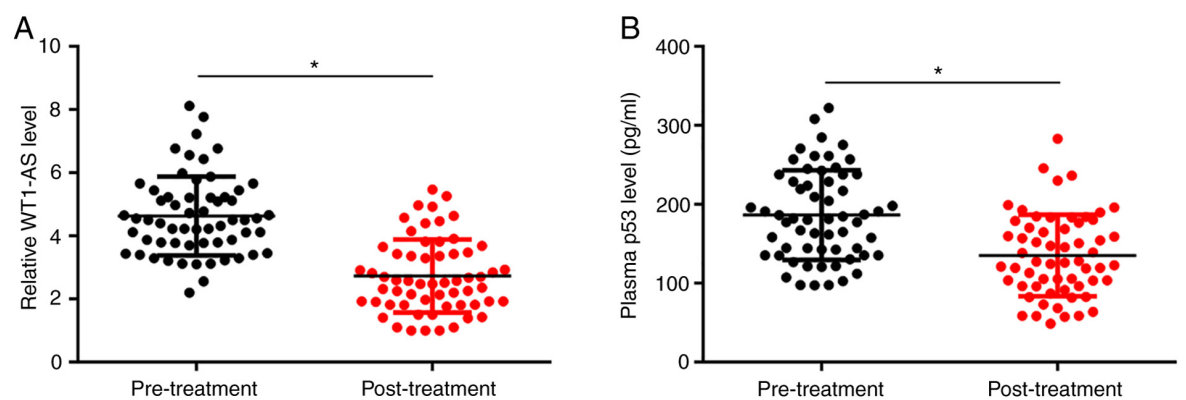

Figure 3. Bisphosphonate therapy downregulates WT1-AS and p53 plasma levels in osteoporosis patients. Plasma levels of (A) WT1-AS and (B) p53 were measured pre-treatment and at 3 months post-treatment. Plasma levels of WT1-AS and p53 were compared between the two time points by performing a paired t-test. ${ }^{*} \mathrm{P}<0.05$. WT1-AS, long non-coding WT1 antisense RNA; p53, cellular tumor antigen $\mathrm{p} 53$.

Altered expression of WT1-AS and p53 separates patients with osteoporosis from healthy controls. The potential application of plasma WT1-AS and p53 for the diagnosis of osteoporosis was explored by performing a ROC curve analysis. The area under the curve (AUC) of plasma WT1-AS was 0.94 (95\% confidence interval, 0.91-0.98; standard error, 0.019; $\mathrm{P}<0.0001$; Fig. 2A), while the AUC of plasma p53 was 0.91 (95\% confidence interval, 0.85-0.96; standard error, 0.026; P<0.0001; Fig. 2B).

Bisphosphonate therapy downregulates WT1-AS and p53 plasma levels in patients with osteoporosis. Plasma levels of WT1-AS and p53 were measured during pre-treatment and at 3 months post-treatment. Levels of WT1-AS (Fig. 3A) and p53 (Fig. 3B) significantly decreased at 3 months post-treatment compared with pre-treatment levels $(\mathrm{P}<0.05)$.

WT1-AS positively regulates $p 53$ expression in osteoblasts. Osteoblasts were transfected with WT1-AS and $\mathrm{p} 53$ expression vectors and WT1-AS siRNA. WT1-AS and p53 expression was significantly altered compared with $\mathrm{NC}$ and $\mathrm{C}$ groups at $24 \mathrm{~h}$ post-transfection $(\mathrm{P}<0.05$; Fig. 4A). Moreover, WT1-AS overexpression resulted in upregulated p53 expression, while WT1-AS siRNA silencing resulted in downregulated p53 expression at mRNA and protein levels compared with the control and $\mathrm{NC}$ groups ( $\mathrm{P}<0.05$; Fig. 4B).

WT1-AS promotes osteoblast apoptosis through p53. WT1-AS and $\mathrm{p} 53$ overexpression resulted in significantly increased apoptosis rates, while WT1-AS siRNA silencing resulted in significantly decreased rates of osteoblast apoptosis compared with $\mathrm{NC}$ and $\mathrm{C}$ groups. In addition, p53 overexpression was indicated to attenuate the effect of WT1-AS siRNA silencing on cell apoptosis in comparison to cells with WT1-AS siRNA transfection alone ( $\mathrm{P}<0.05$; Fig. 5).

\section{Discussion}

To date, the functions of WT1-AS have only been investigated in a few types of cancer $(13,17,18)$. The participation of WT1-AS in cancer biology is mainly mediated by its roles in regulating cell behaviors such as proliferation, apoptosis and invasion $(3,17,16)$. It is known that cell death in osteoblasts contributes to the pathogenesis of osteoporosis (19). The present study investigated the roles of WT1-AS in osteoporosis. WT1-AS was upregulated in osteoporosis and exhibited diagnostic values. In addition, WT1-AS was revealed to serve a role in osteoblast apoptosis and indicated an association with $\mathrm{p} 53$, which may indicate an interaction between the genes. Therefore, overexpression of WT1-AS may promote the progression of osteoporosis by promoting osteoblast apoptosis.

The diagnosis of osteoporosis mainly relies on the measurement of bone mineral density (BMD) (20). However, the threshold of BMD to diagnose osteoporosis is debated, and there is no way to use BMD to screen people with high risk of osteoporosis (20). In the present study, ROC curve analysis indicated that WT1-AS and p53 expression could be used to distinguish patients with osteoporosis from healthy controls. In addition, increased levels of WT1-AS and p53 were observed after treatment with bisphosphonates or estrogen. Therefore, 

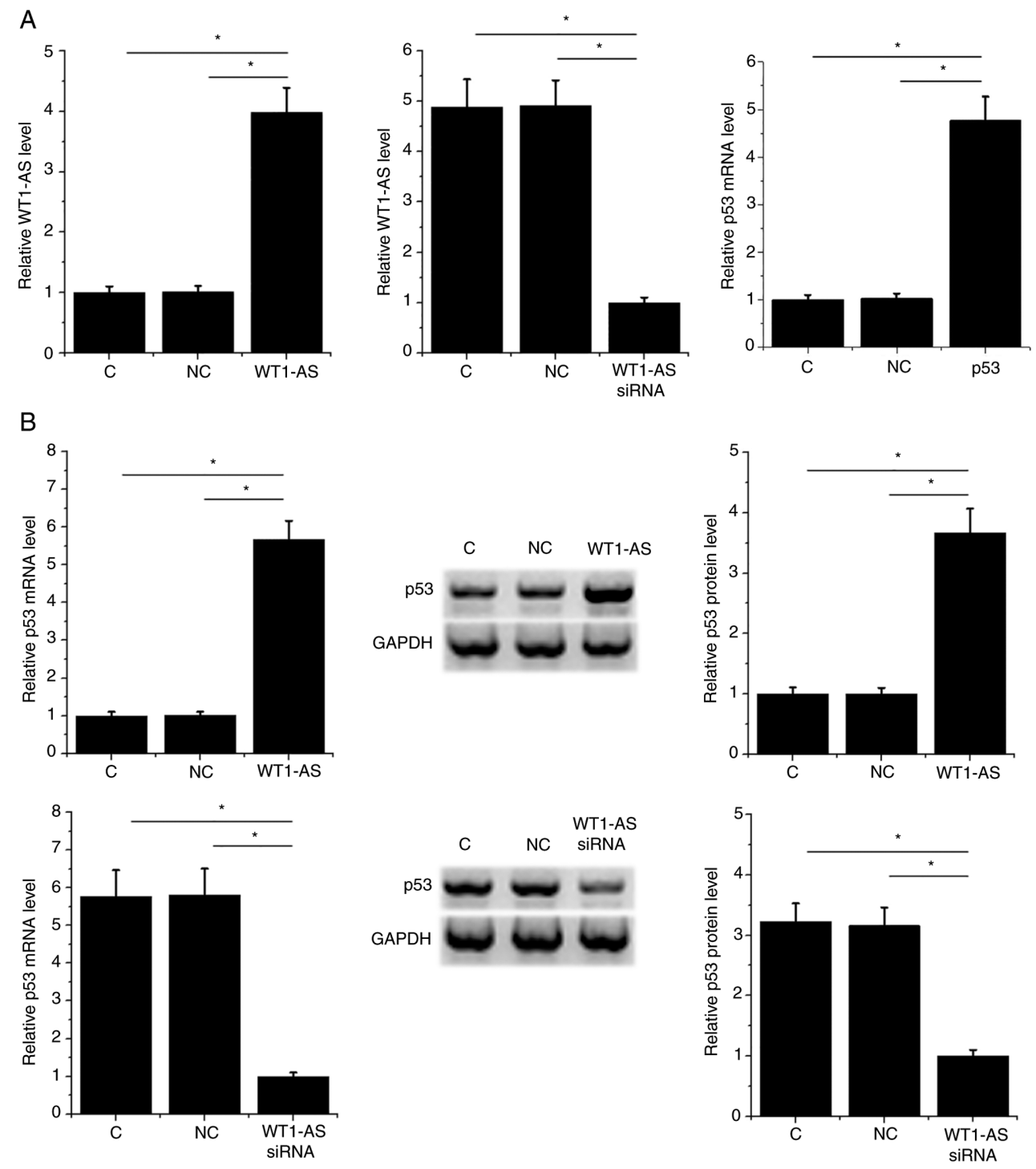

Figure 4. WT1-AS positively regulates p53 expression in osteoblasts. Osteoblasts were transfected with WT1-AS and p53 expression vectors and WT1-AS siRNA. (A) Plasmid and siRNA transfections were confirmed by RT-qPCR analysis. (B) The effects of WT1-AS overexpression and silencing on p53 expression were studied by performing western blotting and RT-qPCR. "P<0.05. siRNA, small interfering RNA; C, control; NC, negative control; RT-qPCR, reverse transcription-quantitative PCR; WT1-AS, long non-coding WT1 antisense RNA; p53, cellular tumor antigen p53.
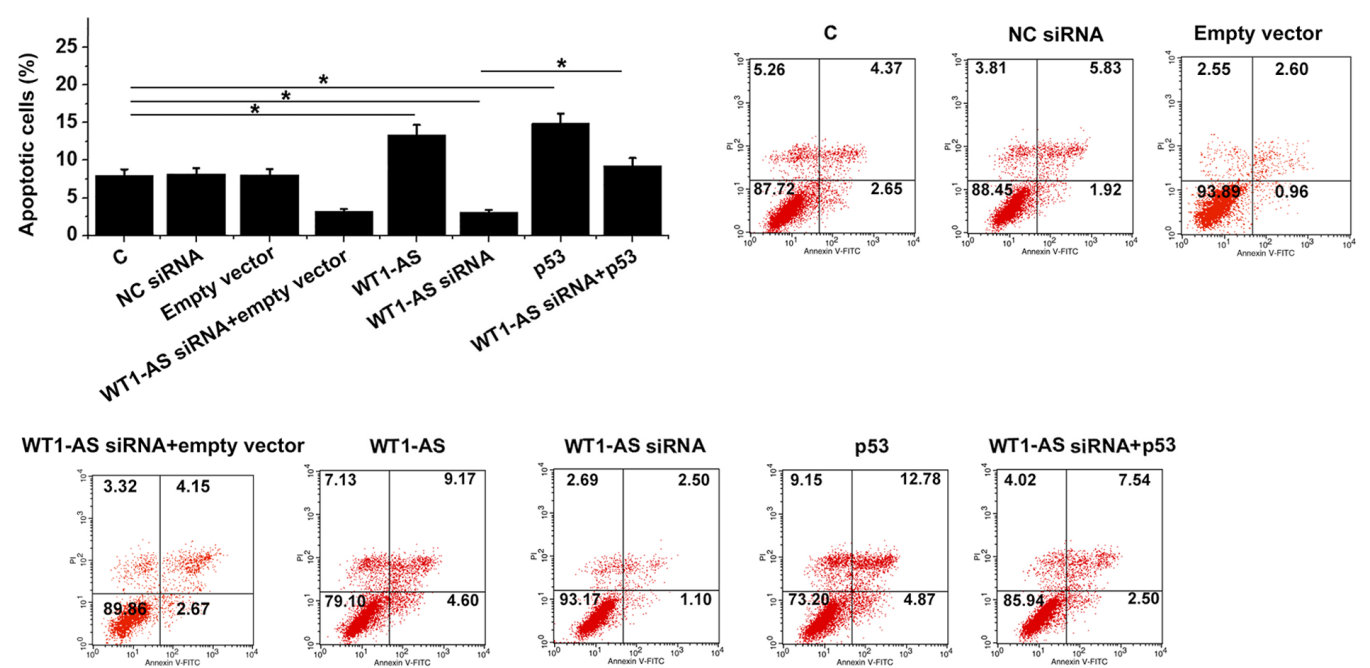

Figure 5. WT1-AS promotes osteoblast apoptosis through p53. The effects of WT1-AS and p53 overexpression as well as WT1-AS siRNA silencing on osteoblast apoptosis were analyzed by performing a cell apoptosis assay. Data among groups were compared by performing one-way ANOVA followed by Tukey's test, "P<0.05. siRNA, small interfering RNA; C, control; NC, negative control; WT1-AS, long non-coding WT1 antisense RNA; p53, cellular tumor antigen p53. 
plasma WT1-AS and p53 may be used as a marker to predict osteoporosis. However, clinical trials are required to test sensitivity and specificity.

The results of the current study revealed that WT1-AS positively regulated the expression of p53 in osteoblasts. The lack of significant correlation between WT1-AS and p53 across the healthy controls suggested that the interaction between them was indirect. It is known that lncRNAs can sponge miRNAs to upregulate the expression of their downstream genes (21). Future studies should assess the involvement of miRNAs and its interaction with lncRNAs. WT1-AS can sponge miR-330-5p to regulate p53 in cervical cancer (13). Future studies should try to explore the involvement of miRNAs in this process.

The present study only investigated the expression of WT1-AS in plasma. Future studies should include patient osteoclasts and animal model experiments to further verify the results of the current study. The pathogenesis of osteoporosis is complicated and requires the involvement of multiple factors, such as epigenetic and hormonal factors (22-27). The interactions between WT1-AS and these factors are needed to be further analyzed.

However, the present study has limitations, including the small sample size. Future studies with bigger sampler sizes are required to further confirm the results of the current study. The involvement of p53-related apoptotic factors, including Bax and Bcl-2, was not explored and p53 knockdown experiments were not included. The current study did not include osteoblast functionality and proliferation assays and the analysis of changes in the expression of marker genes. Therefore, future studies are needed to examine these factors.

In conclusion, WT1-AS was demonstrated to be upregulated in osteoporosis and promoted the apoptosis of osteoblasts by upregulating p53.

\section{Acknowledgements}

Not applicable.

\section{Funding}

This research was supported by the Youth Foundation Project of the First Affiliated Hospital of Hainan Medical College in 2018 (grant no. HYFYPY201813) and Key R\&D Program Projects of Hainan Province in 2018 (grant no. ZDYF2018158).

\section{Availability of data and materials}

The datasets used and/or analyzed during the current study are available from the corresponding author on reasonable request.

\section{Authors' contributions}

CW, QX and YZ designed experiments. CW and QX performed experiments. WS and YL analysed data. YZ drafted the manuscript. All authors read and approved the manuscript.

\section{Ethics approval and consent to participate}

The Ethics Committee of the First Affiliated Hospital of Hainan Medical College approved this study prior to the admission of subjects.

\section{Patient consent for publication}

Not applicable.

\section{Competing interests}

The authors declare they have no competing interests.

\section{References}

1. Siris ES, Adler R, Bilezikian J, Bolognese M, Dawson-Hughes B, Favus MJ, Harris ST, Jan de Beur SM, Khosla S, Lane NE, et al: The clinical diagnosis of osteoporosis: A position statement from the National Bone Health Alliance Working Group. Osteoporos Int 25: 1439-1443, 2014

2. Khosla S and Hofbauer LC: Osteoporosis treatment: Recent developments and ongoing challenges. Lancet Diabetes Endocrinol 5: 898-907, 2017.

3. Lötters FJB, van den Bergh JP, de Vries F and Rutten-van Mölken MP: Current and future incidence and costs of osteoporosis-related fractures in the Netherlands: Combining claims data with BMD measurements. Calcif Tissue Int 98: 235-243, 2016.

4. Curtis EM, Moon RJ, Dennison EM, Harvey NC and Cooper C: Recent advances in the pathogenesis and treatment of osteoporosis. Clin Med (Lond) 15 (Suppl 6): S92-S96, 2015.

5. Raisz LG: Pathogenesis of osteoporosis: Concepts, conflicts, and prospects. J Clin Invest 115: 3318-3325, 2005.

6. Reyes J, Chen JY, Stewart-Ornstein J, Karhohs KW, Mock CS and Lahav G: Fluctuations in p53 signaling allow escape from cell-cycle arrest. Mol Cell. 2018, 71: 581-591. e5, 2018.

7. Willms A, Schittek H, Rahn S, Sosna J, Mert U, Adam D and Trauzold A: Impact of p53 status on TRAIL-mediated apoptotic and non-apoptotic signaling in cancer cells. PLoS One 14: e0214847, 2019.

8. Yeo CQX, Alexander I, Lin Z, Lim S, Aning OA, Kumar R, Sangthongpitag K, Pendharkar V, Ho VHB and Cheok CF: p53 maintains genomic stability by preventing interference between transcription and replication. Cell Rep 15: 132-146, 2016.

9. Hirasawa H, Tanaka S, Sakai A, Tsutsui M, Shimokawa H, Miyata H,Moriwaki S, Niida S, Ito M and Nakamura T: ApoE gene deficiency enhances the reduction of bone formation induced by a high-fat diet through the stimulation of p53-mediated apoptosis in osteoblastic cells. J Bone Miner Res 22: 1020-1030, 2007.

10. Weinstein RS and Manolagas SC: Apoptosis and osteoporosis. Am J Med 108: 153-164, 2000.

11. Zhen YF, Wang GD, Zhu LQ, Tan SP, Zhang FY, Zhou XZ and Wang XD: P53 dependent mitochondrial permeability transition pore opening is required for dexamethasone-induced death of osteoblasts. J Cell Physiol 229: 1475-1483, 2014.

12. Hao L, Fu J, Tian Y and Wu J: Systematic analysis of lncRNAs, miRNAs and mRNAs for the identification of biomarkers for osteoporosis in the mandible of ovariectomized mice. Int J Mol Med 40: 689-702, 2017.

13. Cui L, Nai M, Zhang K, Li L and Li R: IncRNA WT1-AS inhibits the aggressiveness of cervical cancer cell via regulating p53 expression via sponging miR-330-5p. Cancer Manag Res 11: 651-667, 2019.

14. Coulson KA, Reed G, Gilliam BE, Kremer JM and Pepmueller PH: Factors influencing fracture risk, $\mathrm{T}$ score, and management of osteoporosis in patients with rheumatoid arthritis in the Consortium of Rheumatology Researchers of North America (CORRONA) registry. J Clin Rheumatol 15: 155-160, 2009.

15. Al-Azzawi F: Prevention of postmenopausal osteoporosis and associated fractures: Clinical evaluation of the choice between estrogen and bisphosphonates. Gynecol Endocrinol 24: 601-609, 2008.

16. Livak KJ and Schmittgen TD: Analysis of relative gene expression data using real-time quantitative PCR and the 2(-Delta Delta C(T)) Method. Methods 25: 402-408, 2001.

17. Du T, Zhang B, Zhang S, Jiang X, Zheng P, Li J, Yan M, Zhu Z and Liu B: Decreased expression of long non-coding RNA WT1-AS promotes cell proliferation and invasion in gastric cancer. Biochim Biophys Acta 1862: 12-19, 2016.

18. Dai SG, Guo LL, Xia X and Pan Y: Long non-coding RNA WT1-AS inhibits cell aggressiveness via miR-203a-5p/FOXN2 axis and is associated with prognosis in cervical cancer. Eur Rev Med Pharmacol Sci 23: 486-495, 2019. 
19. Komori T: Cell death in chondrocytes, osteoblasts, and osteocytes. Int J Mol Sci 17: 2045, 2016.

20. Lorentzon M and Cummings SR: Osteoporosis: The evolution of a diagnosis. J Intern Med 277: 650-661, 2015.

21. Thomson DW and Dinger ME: Endogenous microRNA sponges: Evidence and controversy. Nat Rev Genet 17: 272-283, 2016.

22. Yu Y, Newman H, Shen L, Sharma D, Hu G, Mirando AJ, Zhang H, Knudsen E, Zhang GF, Hilton MJ, et al: Glutamine metabolism regulates proliferation and lineage allocation in skeletal stem cells. Cell Metab 29: 966-978. e4, 2019.

23. Hayashi M, Nakashima T, Yoshimura N, Okamoto K, Tanaka S and Takayanagi H: Autoregulation of osteocyte Sema3A orchestrates estrogen action and counteracts bone aging. Cell Metab 29: 627-637.e5, 2019.

24. Fan Y, Hanai JI, Le PT, Bi R, Maridas D, DeMambro V, Figueroa CA, Kir S, Zhou X, Mannstadt M, et al: Parathyroid hormone directs bone marrow mesenchymal cell fate. Cell Metab 25: 661-672, 2017.
25. Stegen S, van Gastel N, Eelen G, Ghesquière B, D'Anna F, Thienpont B, Goveia J, Torrekens S, Van Looveren R, Luyten FP, et al: HIF-1 $\alpha$ promotes glutamine-mediated redox homeostasis and glycogen-dependent bioenergetics to support postimplantation bone cell survival. Cell Metab 23: 265-279, 2016.

26. Liu S, Liu D, Chen C, Hamamura K, Moshaverinia A, Yang R, Liu Y, Jin Y and Shi S: MSC transplantation improves osteopenia via epigenetic regulation of notch signaling in lupus. Cell Metab 22: 606-618, 2015.

27. Mera P, Laue K, Ferron M, Confavreux C, Wei J, Galán-Díez M, Lacampagne A, Mitchell SJ, Mattison JA, Chen Y, et al: Osteocalcin signaling in myofibers is necessary and sufficient for optimum adaptation to exercise. Cell Metab 23: 1078-1092, 2016.

(i) $($ ) This work is licensed under a Creative Commons Attribution-NonCommercial-NoDerivatives 4.0 International (CC BY-NC-ND 4.0) License. 